\title{
Protocol
}

\section{Determination of Gross Chromosomal Rearrangement Rates}

\author{
Christopher D. Putnam ${ }^{1,2,6}$ and Richard D. Kolodner ${ }^{1,2,3,4,5}$ \\ ${ }^{1}$ Ludwig Institute for Cancer Research, La Jolla, CA 92093-2385, USA \\ ${ }^{2}$ Department of Medicine, University of California, La Jolla, CA 92093, USA \\ ${ }^{3}$ Department of Cellular and Molecular Medicine, San Diego School of Medicine, University of California, La Jolla, \\ CA 92093, USA \\ ${ }^{4}$ Moores Cancer Center, San Diego Medical Center, University of California, La Jolla, CA 92093, USA \\ ${ }^{5}$ Institute of Genomic Medicine, San Diego Health Sciences, University of California, La Jolla, CA 92093, USA
}

[Supplemental material is available at http://www.cshprotocols.org/supplemental/.]

\section{INTRODUCTION}

Cells devote a significant amount of metabolism to maintaining the stability of their genome and to preventing inappropriate chromosomal rearrangements that are characteristic of many cancers. A simple genetic assay using haploid derivatives of the yeast Saccharomyces cerevisiae provides a means to quantitatively measure the rate at which gross chromosomal rearrangements (GCRs) accumulate in different genetic backgrounds. This assay measures the rate of simultaneous inactivation of CAN1 and URA3 markers placed on a nonessential end of a yeast chromosome and in principle can be implemented in any haploid strain. Rearrangements detected with this assay include broken chromosomes healed by de novo telomere additions and a spectrum of inter- and intrachromosomal fusion events. The GCR assay allows for detailed analysis of the contributions of individual genes and different pathways in the suppression of genomic instability.

\section{MATERIALS}

CAUTIONS AND RECIPES: Please see Appendices for appropriate handling of materials marked with $\langle$ ! $>$, and recipes for reagents marked with $<\mathbf{R}>$.

\section{Reagents}

$<\mathbf{R}><$ ! $>$ 5-FOA Can agar plate media

S. cerevisiae strains appropriate for GCR measurements (e.g., RDKY3615 [MATa ura3-52 leu2 $\Delta 1$, trp1 $\Delta 63$ his3 $\Delta 200$ lys2 $\Delta B g$ l hom3-10 ade2 $\Delta 1$ ade8 hxt13::URA3]; RDKY6677 [MATa ura3-52 leu2 $\Delta 1$, trp1 $\Delta 63$ his3 $\Delta 200$ lys2 $\Delta B g$ l hom3-10 ade2 $\Delta 1$ ade8 yel068c::CAN1/URA3 iYEL072W::hph]; and RDKY6678 [MATa ura3-52 leu2 $\Delta 1$, trp1 $\Delta 63$ his3 200 lys 2 $\Delta B g l$ hom3-10 ade2 11 ade8 yel072w::CAN1/URA3 iYEL072W::hph])

$<\mathrm{R}>$ YPD media

Prepare both liquid media and agar plates.

\section{Equipment}

Centrifuge

Culture flasks, sterile (of at least twice the volume of the culture; see Step 2)

${ }^{6}$ Corresponding author (cdputnam@ucsd.edu). 


\section{METHOD}

\section{GCR Fluctuation Analysis}

1. Streak the yeast strains of interest onto YPD agar plates. Use at least two independent biological isolates per strain.

Colonies should be visible in 2-3 $d$ if the strains have no growth defects and are grown on rich media. It is important to have well-separated single colonies. Strains with variable-sized colonies should be carefully investigated. Smaller colonies can indicate the spontaneous generation of respiratory-defective petites, whereas larger colonies can indicate the presence of spontaneous mutations that suppress growth defects caused by the desired genotype. Ideally, similar-sized colonies that lack additional genetic changes should be analyzed.

2. Depending on the expected GCR rate, choose an appropriate volume of YPD liquid medium for growing liquid cultures:

i. For strains with rates similar to wild type $\left(\sim 10^{-10}\right.$ mutations per cell per generation), use 50-mL liquid cultures.

ii. For moderately increased GCR rates ( $10^{-8}$ mutations per cell per generation), use $10-\mathrm{mL}$ liquid cultures.

iii. For very high GCR rates $\left(\sim 10^{-6}\right.$ mutations per cell per generation), use 1 - to 5 -mL liquid cultures.

iv. For unknown rates, initially try 10-mL liquid cultures.

3. For each culture, use a sterile scalpel blade to cut out one colony from the Petri dish. Inoculate each liquid culture with a single colony/agar plug.

Ensure that the entire colony is used in the inoculation and that each colony/agar plug is not contaminated with cells from other colonies. Grow at least seven cultures for each independent biological isolate; larger numbers of cultures allow more accurate determination of rates.

4. Grow cultures at $30^{\circ} \mathrm{C}$ with vigorous shaking until they reach saturation.

In rich media, saturation typically occurs after 24-36 h of growth for strains with wild-type growth rates. Sicker strains can take longer to reach saturation and can reach saturation at lower viable cell densities than wild-type strains; determination of experimental growth curves for these types of strains can be helpful.

5. For each culture, label five sterile microcentrifuge tubes A-E. Add $90 \mu \mathrm{L}$ of sterile water to each.

6. Prepare a $10^{-5}$ dilution for each culture as follows:

i. Transfer $10 \mu \mathrm{L}$ of the culture to tube $\mathrm{A}$ and vortex.

ii. Transfer $10 \mu \mathrm{L}$ of $\mathrm{A}$ to $\mathrm{B}$ and vortex.

iii. Transfer $10 \mu \mathrm{L}$ of $\mathrm{B}$ to $\mathrm{C}$ and vortex.

iv. Transfer $10 \mu \mathrm{L}$ of $\mathrm{C}$ to $\mathrm{D}$ and vortex.

v. Transfer $10 \mu \mathrm{L}$ of $\mathrm{D}$ to $\mathrm{E}$ and vortex.

7. Spread all $100 \mu \mathrm{L}$ of each tube $\mathrm{E}$ (i.e., the $10^{-5}$ dilution) on 9-cm YPD agar plates. This provides a final effective dilution of $10^{-6}$ viable cells per milliliter, relative to the concentration of the culture.

8. Centrifuge the remainder of the saturated culture at $2000 \mathrm{~g}$ for $10 \mathrm{~min}$ at room temperature. Carefully decant the supernatant. 
9. Resuspend the pellet in $0.2 \mathrm{~mL}$ of sterile water per $10 \mathrm{~mL}$ of original culture.

10. Spread the resuspended culture onto $14.5-\mathrm{cm} 5$-FOA Can plates.

For up to $10 \mathrm{~mL}$ of culture $\left(\sim 10^{9}\right.$ cells), one $14.5-\mathrm{cm}$ plate can be used. For larger cultures, divide each culture evenly between multiple plates, plating no more than $10^{9}$ cells/plate for each culture.

11. Place the YPD and 5-FOA Can plates in the incubator at $30^{\circ} \mathrm{C}$.

Colonies with wild-type growth rates should take 3-5 d to appear on 5-FOA Can plates.

12. Count the number of colonies on the YPD and 5-FOA Can plates. In rich media, wild-type cultures at saturation have $\sim 10^{8}$ cells $/ \mathrm{mL}$, yielding $\sim 100$ colonies $/ Y P D$ plate. See Troubleshooting.

\section{GCR Rate Calculations}

13. To calculate the number of viable cells per milliliter $(D)$ for each culture, multiply the number of colonies on the YPD plate by the total dilution factor (in this case, $10^{6}$; see Steps 6 and 7).

14. For each culture, calculate the number of viable cells in the culture $(N)$ using the formula $N=D \times$ $V$, where $V$ is the volume of the culture (in milliliters), and $D$ is the viable cells per milliliter (from Step 13).

15. For each culture, estimate the most likely number of mutational events $(m)$ using the observed number of colonies on the 5-FOA Can plates $(r)$ and the equation $m(1.24+\ln [m])-r=0$.

This equation was originally derived by Lea and Coulson (1949). Values of $\mathrm{r}$ as a function of $\mathrm{m}$ can readily be tabulated in spreadsheets (Table 1, or $\mathrm{m}$ can be calculated from $\mathrm{r}$ by iterative methods (e.g., bisection, NewtonRaphson). A Visual Basic macro for calculating $\mathrm{m}$ from $\mathrm{r}$ is available as Supplemental Material (Suppl_RtoM_Conversion_Macro.doc).

16. For each culture, calculate $P$, the most likely rate (in events per cell per generation) using the formula $P=m / N$, where $N$ is the number of cells in the culture (see Step 14) and $m$ is the most likely number of events (from Step 15).

17. For each biological isolate, order all the most likely rates ( $P$, from Step 16) from smallest to largest, including all zero rates.

18. Determine the median rate of the cultures, $R$.

The median is the value of the middle element in the ordered list (or the average of the two middle values if there are an even number of elements in the list).

19. If $R=0$, calculate an upper limit for the rate $(R)$ by replacing all cultures with $r=0$ with $r=1$ and determining the new median of these rates. Report the resulting rate as being less than $R^{\prime}$. For example, if $R=0$ and $R^{\prime}=1.75 \times 10^{-9}$, the reported rate should be $<1.75 \times 10^{-9}$.

20. Determine the $95 \%$ confidence intervals for the median (see, e.g., http://www.math.unb.ca/ $\sim$ knight/utility/Medlnt95.htm). For six to 283 cultures, calculate the lower $(L)$ and upper $(U) 95 \%$ confidence interval limits using the equations $L=$ floor $[(M+1) / 2-0.9789 \sqrt{M}]$ and $U=(M+1)-$ $L$, where $M$ is the number of cultures.

Briefly, the lower and upper $95 \%$ confidence interval limits correspond to the $L$-th and $U$-th positions in the ordered list of individual rates. For example, if the number of cultures $(M) 14, \mathrm{~L}=$ floor $[15 / 2-0.9789 \sqrt{14}]=3 ; U=(14+1)-3=12$. Thus, the $95 \%$ confidence interval is defined by the third and 12 th lowest mutation rates.

See Troubleshooting.

21. If the median rates for the different biological isolates are within the $95 \%$ confidence intervals, merge all rates from the different biological isolates. Determine the median rate and the $95 \%$ confidence intervals for the combined list of rates (i.e., repeat Steps 17-20 for the entire set of rates from all biological isolates of a given genotype). 
Downloaded from http://cshprotocols.cshlp.org/ on April 26, 2023 - Published by Cold Spring Harbor Laboratory Press

Table 1. $r$ and $m$ values

\begin{tabular}{|c|c|c|c|}
\hline$r$ & $m$ & $r$ & $m$ \\
\hline 0 & 0.000000 & 51 & 13.318792 \\
\hline 1 & 0.890054 & 52 & 13.525536 \\
\hline 2 & 1.318699 & 53 & 13.731630 \\
\hline 3 & 1.696365 & 54 & 13.937089 \\
\hline 4 & 2.045410 & 55 & 14.141929 \\
\hline 5 & 2.375203 & 56 & 14.346164 \\
\hline 6 & 2.690785 & 57 & 14.549807 \\
\hline 7 & 2.995255 & 58 & 14.752872 \\
\hline 8 & 3.290694 & 59 & 14.955372 \\
\hline 9 & 3.578578 & 60 & 15.157320 \\
\hline 10 & 3.860007 & 61 & 15.358725 \\
\hline 11 & 4.135825 & 62 & 15.559601 \\
\hline 12 & 4.406700 & 63 & 15.759959 \\
\hline 13 & 4.673170 & 64 & 15.959807 \\
\hline 14 & 4.935678 & 65 & 16.159158 \\
\hline 15 & 5.194593 & 66 & 16.358019 \\
\hline 16 & 5.450227 & 67 & 16.556402 \\
\hline 17 & 5.702849 & 68 & 16.754315 \\
\hline 18 & 5.952690 & 69 & 16.951767 \\
\hline 19 & 6.199949 & 70 & 17.148766 \\
\hline 20 & 6.444805 & 71 & 17.345321 \\
\hline 21 & 6.687414 & 72 & 17.541440 \\
\hline 22 & 6.927914 & 73 & 17.737130 \\
\hline 23 & 7.166431 & 74 & 17.932399 \\
\hline 24 & 7.403076 & 75 & 18.127254 \\
\hline 25 & 7.637951 & 76 & 18.321702 \\
\hline 26 & 7.871146 & 77 & 18.515750 \\
\hline 27 & 8.102747 & 78 & 18.709405 \\
\hline 28 & 8.332830 & 79 & 18.902672 \\
\hline 29 & 8.561464 & 80 & 19.095559 \\
\hline 30 & 8.788715 & 81 & 19.288071 \\
\hline 31 & 9.014641 & 82 & 19.480214 \\
\hline 32 & 9.239299 & 83 & 19.671994 \\
\hline 33 & 9.462740 & 84 & 19.863417 \\
\hline 34 & 9.685011 & 85 & 20.054487 \\
\hline 35 & 9.906156 & 86 & 20.245211 \\
\hline 36 & 10.126218 & 87 & 20.435592 \\
\hline 37 & 10.345235 & 88 & 20.625637 \\
\hline 38 & 10.563242 & 89 & 20.815350 \\
\hline 39 & 10.780276 & 90 & 21.004736 \\
\hline 40 & 10.996367 & 91 & 21.193799 \\
\hline 41 & 11.211547 & 92 & 21.382545 \\
\hline 42 & 11.425843 & 93 & 21.570976 \\
\hline 43 & 11.639283 & 94 & 21.759098 \\
\hline 44 & 11.851892 & 95 & 21.946915 \\
\hline 45 & 12.063695 & 96 & 22.134431 \\
\hline 46 & 12.274714 & 97 & 22.321649 \\
\hline 47 & 12.484972 & 98 & 22.508575 \\
\hline 48 & 12.694490 & 99 & 22.695210 \\
\hline 49 & 12.903287 & 100 & 22.881560 \\
\hline 5 & 13.111382 & & \\
\hline
\end{tabular}




\section{TROUBLESHOOTING}

Problem: No colonies are observed on the 5-FOA Can plates.

[Step 12]

Solution: Use larger culture sizes and/or test the plates to ensure they support the growth of a can1 ura3 double mutant strain.

Problem: The biological isolates are significantly different on the basis of the $95 \%$ confidence intervals. [Step 20]

Solution: Troubleshooting will depend on the precise genotypes of the strains involved. Consider the following:

1. Test additional biological isolates.

2. Verify strain genotypes.

3. Carefully examine growth rates and drug sensitivities.

4. If need be, construct new isolates for testing.

\section{DISCUSSION}

The genetic assay described here measures the rate of simultaneous inactivation of the CAN1 and URA3 genes in haploid cells of S. cerevisiae using fluctuation analysis (Lea and Coulson 1949). For wild-type cells, this 5-FOA ${ }^{R} C A N^{R}$ rate is the result of chromosomal rearrangements and not simultaneous inactivation of URA3 and CAN1 by point mutations. However, simultaneous inactivation of URA3 and CAN1 by point mutations has been observed in mismatch repair-defective strains (Myung et al. 2001a). The original assay strain (RDKY3615) contains a hxt13::URA3 insertion telomeric to CAN1 on the nonessential region of the left arm of chromosome $V$ (Chen and Kolodner 1999). Several modified versions of the assay have since been developed, including those with a CAN1/URA3 cassette placed at a number of different chromosomal locations (RDKY6677, RDKY6678) (Putnam et al. 2009), as well as other variant GCR assays using the loss of URA3 and HIS3 markers on chromosome III (Myung et al. 2001b) or loss of CAN1, URA3, and ADE2 on chromosome XV (Hackett et al. 2001).

The observed range of chromosome $\mathrm{V}$ alterations includes broken chromosomes healed by de novo telomere addition and various forms of translocations and other genome rearrangements that are similar to many of the rearrangements observed in human cancers (Mitelman 1991). These translocations include monocentric translocations, telomere capture events, and interstitial deletions as well as dicentric inter- and intrachromosomal fusions; the dicentric products appear to be unstable and undergo additional rearrangements resulting in stable monocentric products (Pennaneach and Kolodner 2009). Analysis of products can involve polymerase chain reaction (PCR)-based mapping and breakpoint sequencing (Chen and Kolodner 1999; Smith et al. 2004; Schmidt et al. 2006a), PCR amplification of predicted breakpoint junctions (Schmidt et al. 2006b; Motegi and Myung 2007; Putnam et al. 2009), linker-mediated PCR (Motegi and Myung 2007), clamped homogeneous electric field gel analysis (Pennaneach and Kolodner 2009), and array competitive genomic hybridization experiments (Pennaneach and Kolodner 2009; Putnam et al. 2009).

The GCR rates and the resulting chromosomal rearrangement products observed are dependent on a number of factors, including the genomic features present between the counter-selectable markers and the nearest essential genes (Putnam et al. 2009) and the strain genotypes (Chen and Kolodner 1999; Kolodner et al. 2002). This simple protocol for determining GCR mutation rates in a variety of genetic backgrounds coupled with a diversity of modified GCR assays has provided tremendous insight into the large numbers of pathways that suppress genomic instability in S. cerevisiae and appear to be relevant to cancer suppression pathways in humans (Kolodner et al. 2002; Wang et al. 2005).

\section{REFERENCES}

Chen C, Kolodner RD. 1999. Gross chromosomal rearrangements in Saccharomyces cerevisiae replication and recombination defective mutants. Nat Genet 23: 81-85.

Hackett JA, Feldser DM, Greider CW. 2001. Telomere dysfunction increases mutation rate and genomic instability. Cell 106: 275-286.

Kolodner RD, Putnam CD, Myung K. 2002. Maintenance of genome stability in Saccharomyces cerevisiae. Science 297: 552-557. 
Lea DE, Coulson CA. 1949. The distribution of the number of mutants in bacterial populations. J Genet 49: 264-285.

Mitelman F. 1991. Catalog of chromosomal aberrations in cancer, 4th ed. Wiley-Liss, New York.

Motegi A, Myung K. 2007. Measuring the rate of gross chromosomal rearrangements in Saccharomyces cerevisiae: A practical approach to study genomic rearrangements observed in cancer. Methods 41: 168-176.

Myung K, Datta A, Chen C, Kolodner RD. 2001a. SGS1, the Saccharomyces cerevisiae homologue of BLM and WRN, suppresses genome instability and homeologous recombination. Nat Genet 27: 113-116.

Myung K, Datta A, Kolodner RD. 2001b. Suppression of spontaneous chromosomal rearrangements by $S$ phase checkpoint functions in Saccharomyces cerevisiae. Cell 104: 397-408.

Pennaneach V, Kolodner RD. 2009. Stabilization of dicentric translocations through secondary rearrangements mediated by multiple mechanisms in S. cerevisiae. PLoS ONE 4: e6389. doi: 10.1371/ journal.pone.0006389.
Putnam CD, Hayes TK, Kolodner RD. 2009. Specific pathways prevent duplication-mediated genome rearrangements. Nature 460: 984-989.

Schmidt KH, Pennaneach V, Putnam CD, Kolodner RD. 2006 a. Analysis of gross-chromosomal rearrangements in Saccharomyces cerevisiae. Methods Enzymol 409: 462-476.

Schmidt KH, Wu J, Kolodner RD. 2006b. Control of translocations between highly diverged genes by Sgs1, the Saccharomyces cerevisiae homolog of the Bloom's syndrome protein. Mol Cell Biol 26: 5406-5420.

Smith S, Hwang J-Y, Banerjee S, Majeed A, Gupta A, Myung K. 2004. Mutator genes for suppression of gross chromosomal rearrangements identified by genome-wide screening in Saccharomyces cerevisiae. Proc Natl Acad Sci 101: 9039-9044.

Wang Y, Putnam CD, Kane MF, Zhang W, Edelmann L, Russell R, Carrión DV, Chin L, Kucherlapati R, Kolodner RD, et al. 2005. Mutation in Rpa1 results in defective DNA double-strand break repair, chromosomal instability and cancer in mice. Nat Genet 37: 750-755. 


\section{Determination of Gross Chromosomal Rearrangement Rates}

Christopher D. Putnam and Richard D. Kolodner

Cold Spring Harb Protoc; doi: 10.1101/pdb.prot5492

\begin{tabular}{|c|c|}
\hline $\begin{array}{l}\text { Email Alerting } \\
\text { Service }\end{array}$ & Receive free email alerts when new articles cite this article - click here. \\
\hline $\begin{array}{l}\text { Subject } \\
\text { Categories }\end{array}$ & $\begin{array}{l}\text { Browse articles on similar topics from Cold Spring Harbor Protocols. } \\
\text { Bioinformatics/Genomics, general (192 articles) } \\
\text { Genetic Variation ( } 86 \text { articles) } \\
\text { Genetics, general ( } 374 \text { articles) } \\
\text { Genome Analysis (191 articles) } \\
\text { Laboratory Organisms, general (923 articles) } \\
\text { Molecular Biology, general (1293 articles) } \\
\text { Yeast (288 articles) } \\
\text { Yeast Genetics (135 articles) }\end{array}$ \\
\hline
\end{tabular}

